$\begin{array}{cl}\begin{array}{cl}\text { Revue } \\ \text { de /histoire } \\ \text { des religions }\end{array} & \text { Revue de l'histoire des religions } \\ & 2 \text { Religion, secret et autorité }\end{array}$

\title{
La langue du silence dans le Maroc urbain contemporain
}

The Language of Silence in Contemporary Urban Morocco

\section{Anouk Cohen}

\section{(2) OpenEdition}

12 Journals

Édition électronique

URL : http://journals.openedition.org/rhr/7779

DOI : $10.4000 /$ rhr.7779

ISSN : 2105-2573

Éditeur

Armand Colin

Édition imprimée

Date de publication : 1 juin 2011

Pagination : 245-263

ISBN : 978-2200-92720-2

ISSN : 0035-1423

Référence électronique

Anouk Cohen, "La langue du silence dans le Maroc urbain contemporain », Revue de l'histoire des religions [En ligne], 2 | 2011, mis en ligne le 01 juin 2015, consulté le 19 avril 2019. URL : http:// journals.openedition.org/rhr/7779; DOI : 10.4000/rhr.7779 


\section{ANOUK COHEN}

Université Paris Ouest - Nanterre La Défense

(Laboratoire d'ethnologie et de sociologie comparative, UMR 7186)

\section{La langue du silence dans le Maroc urbain contemporain}

Quatre mois après son lancement en août 2006, l'hebdomadaire arabophone Nichane est interdit par décision du Premier ministre à la suite d'un dossier - en traduction française - intitulé : "Blagues : comment les Marocains rient de la religion, du sexe et de la politique ». Un thème qui avait déjà fait l'objet d'un article en français n'ayant entraîné aucune sanction. Est-ce alors le fait d'avoir rapporté ces blagues en arabe qui soulève la polémique? "L'affaire Nichane " interroge le statut de la langue arabe et de la langue française en milieu urbain marocain par rapport à la religion et à la politique. Est-ce à dire que la langue rend ces sujets tabous? La langue arabe impose-t-elle le silence? Nous interrogerons les liens entre les notions de silence et de secret.

\section{The Language of Silence in Contemporary Urban Morocco}

Four months after its first publication in August 2006, the Arabic weekly newspaper Nichane was banned by a decision of the Prime Minister following a report called (in translation): "Jokes: How Moroccans Laugh at Religion, Sex and Politics". This subject had already been the object of an article in French that had incurred no penalty. Was it thus the fact of having reported these jokes in Arabic that was at the heart of the debate? The "Nichane affair" raises questions about the statuses of the Arabic and French languages in relation to religion and politics in urban Morocco. Does this mean that it is the language that renders these subjects taboo? Does Arabic impose silence? This paper examines the how the notions of silence and secrecy are connected. 
Quatre mois après son lancement en août 2006, l'hebdomadaire arabophone Nichane - écrit en arabe classique et dialectal - est interdit par décision du Premier ministre à la suite de la publication d'un dossier intitulé - en traduction française : «Blagues : comment les Marocains rient de la religion, du sexe et de la politique. ». La volonté de Driss Ksikès, rédacteur en chef du magazine poursuivi pour « atteinte aux valeurs sacrées » et « publication et distribution d'écrits contraires à la morale et aux mœurs », était de retranscrire les blagues «telles qu'elles se racontent», dans la langue dans laquelle elles sont dites, la darija, le dialecte marocain ${ }^{1}$. Ces plaisanteries avaient déjà fait l'objet d'un article en français qui n'avait entraîné aucune sanction lors de sa publication dans le magazine Tel Quel, appartenant au même groupe de presse que Nichane. Estce alors le fait d'avoir rapporté ces blagues en arabe qui a été le point névralgique de la polémique? Est-ce à dire que la langue rend ces sujets tabous? Pourquoi cela a-t-il fait scandale?

On peut considérer le scandale comme « un mode privilégié pour accéder à la réalité sociohistorique d'une population ${ }^{2}$ », un révélateur des rapports de force, des structures ou des normes qui lui préexistent. Damien de Blic et Cyril Lemieux lui attribuent en effet « la capacité de rendre manifeste à l'observateur des lignes de clivage et les rapports de domination qui traversent de manière ordinairement opaque une société ou certaines fractions de ses élites ${ }^{3} »$. Selon Élisabeth Claverie, un scandale donne lieu à une affaire lorsque « le dénonciateur fait à son tour l'objet d'une accusation de la part de l'accusé ou de ses alliés $»^{4}$. C'est dans cette perspective que cet article se propose d'interroger «l'affaire Nichane ». Pour Éric de

1. Durant mon enquête ethnologique effectuée à Casablanca au cours de l'année 2006-2007 dans le cadre d'une recherche doctorale en cours, j'ai pu rencontrer et interroger les auteurs du dossier. Parallèlement à ces entretiens, ma présence permanente sur le terrain m'a permis d'observer les répercussions et les enjeux sociaux, culturels et politiques de l'affaire Nichane.

2. Cyril Lemieux, Damien De Blic, «Le scandale comme épreuve. Éléments de sociologie pragmatique », À l'épreuve du scandale, Revue Politix, 71/2005, p. 9-38.

3. Ibid., p. 12.

4. Ibid., p. 17. 
Dampierre ${ }^{5}$, le scandale implique nécessairement une dimension de secret ou de dissimulation et n'éclate qu'en vertu d'un processus de mise en visibilité et de publication, lorsque la transgression censée rester cachée est soudainement dévoilée. Nous tenterons de saisir ici quel interdit le magazine Nichane est accusé d'avoir enfreint : pour quelles raisons la version arabe a-t-elle été condamnée? Quel impact a eu la mise par écrit de ces plaisanteries dans la langue nationale de communication orale, la darija? Enfin, quel enjeu recouvre l'éclatement de cette « affaire » dans la société marocaine et quelle articulation existe-t-il entre les notions d' « affaire », de « scandale »? Autant d'interrogations qui sont au cœur de cette réflexion axée sur les efforts entrepris par des journalistes pour traiter en arabe de sujets qui, au Maroc - principalement la religion et la politique - sont habituellement couverts par le silence. Aussi, nous nous interrogerons sur les liens entre la notion de silence et la notion de secret.

\section{DU SENS CACHÉ DE LA PRATIQUE SUBVERSIVE}

\section{Traduire « l'intraduisible» : sens secret}

Il est incontournable d'étudier le rôle joué par le processus de traduction dans cette " affaire ». Celui-ci, comme on le sait, constitue le passage d'une langue à une autre et d'une culture à une autre. Traduire de façon fidèle ne consiste pas tant en la quête du « bon » mot censé rendre compte d'une idée, qu'en la recherche de la manière d'exprimer celle-ci'. Là se trouve toute la difficulté de la tâche qui incombe au traducteur qui, plus qu'un passeur de mots, représente un passeur du sens et du sensible. Traduire une langue implique l'accès à la culture correspondante; car une langue vit et évolue à l'instar de la société qui la pratique. La dimension comparatiste est alors essentielle : on ne parle correctement dans une langue que si l'on s'aperçoit qu'il y en a d'autres. La difficulté réside dans le fait que la langue de culture ne cesse de s'élaborer ${ }^{7}$. Il est donc ardu de la

5. Éric De Dampierre, «Thèmes pour l'étude du scandale», Annales, 10/1954, p. 328-336.

6. Walter Benjamin, Euvres, t. I, Paris, Gallimard, 2000, p. 244-262.

7. Barbara Cassin, Vocabulaire européen des philosophies: dictionnaire des intraduisibles, Paris, Seuil, 2004. 
saisir, d'en figer le sens pour le traduire dans une langue autre, ellemême en mouvement. Quand on traduit, on se heurte au signifiant, à la manière dont il résonne, ce que Derrida nomme « l'intraduisible corps des langues ${ }^{8} »$. Un problème aggravé lorsqu'il s'agit de vouloir communiquer une forme d'esprit en prise avec une certaine réalité sociale, comme l'humour. De cette complexité, inhérente à la traduction, il résulte qu'elle est souvent imparfaite. Retranscrites en français, les blagues qui nous intéressent ne rendent pas tout à fait compte du sens entier qu'elles renferment : une part de leur sens s'est perdue lors de la traduction. Dans quelle mesure est-ce précisément cette marge cachée, impénétrable, qui a autorisé les blagues à circuler en français plutôt qu'en arabe? C'est là un questionnement à mettre en relation avec la place respective que tiennent la langue française et la langue arabe au Maroc.

Durant des siècles, la langue arabe a été au Maroc la langue écrite quasi unique, maintenue en même temps que des langues orales dites dialectales (arabes - la darija - ou berbères - l'amazigh). La colonisation (1912-1956) a modifié cette situation par l'utilisation prédominante de la langue française à l'écrit. Les théoriciens de l'impérialisme français étaient alors convaincus que la meilleure infiltration du pays colonisé passait par l'usage de la langue et la diffusion de la culture françaises. Sous le protectorat, l'usage de l'arabe classique fut limité dans le système d'enseignement aux matières religieuses et littéraires et, dans la vie publique, à des secteurs restreints de l'administration locale et judiciaire. L'espace de modernité, principalement administrative, que la colonisation créa à son profit fut réservé à la langue française. Depuis, celle-ci demeure profondément ancrée dans la société marocaine en dépit de la politique d'arabisation menée par Hassan II (1961-1999). Aujourd'hui encore, la langue française exerce une double influence sur la société marocaine : d'un point de vue institutionnel, elle représente la seconde langue de l'enseignement public et de l'administration; d'un point de vue idéologique, elle apparaît comme le signe d'une promotion sociale'. L'histoire

8. Jacques Derrida, Le Monolinguisme de l'autre, Paris, Galilée, 1996.

9. Gilbert Grandguillaume, "Langue arabe et État moderne au Maghreb ", in Jean-Robert Henry (dir.), Nouveaux enjeux culturels au Maghreb, Paris, CNRS, 1986, p. 79-88; Mustapha Haddab, « Arabisation de l'enseignement des sciences et mutations dans le champ socio-linguistique en Algérie », in J.-R. Henry (dir.), Nouveaux Enjeux culturels au Maghreb, op. cit., p. 99-108 
même du pays est à l'origine d'un bilinguisme toujours effectif, au sein duquel la langue française et la langue arabe correspondent à des pratiques et à des représentations différentes.

\section{Langue arabe : langue de la Révélation}

L'histoire de la langue arabe est marquée par le phénomène de diglossie qui définit la coexistence de plusieurs variantes d'une même langue, une sorte de langue à plusieurs étages correspondant à l'usage qu'en font différents groupes sociaux ${ }^{10}$. On distingue le plus souvent deux grandes catégories : la langue parlée ou langue populaire - la darija au Maroc - et la langue écrite ou savante l'arabe classique dit fus 'ha. Celle-ci constitue aujourd'hui la langue officielle du pays. En tant que langue du Coran et de la religion musulmane, l'arabe fus' $h a$ revêt un caractère particulier. Elle est la langue de la Révélation d'Allah à l'ange Gabriel, un statut qui lui confère une dimension singulière tant à l'écrit qu'à l'oral. Contrairement au projet qui a consisté à faire passer l'hébreu d'une langue divine à une langue quotidienne, en la dé-fondamentalisant et en la débarrassant de sa charge religieuse ${ }^{11}$, l'arabe fus'ha constitue toujours la langue de Dieu. À la suite de l'Égypte, le Maroc a déjoué la partition stricte entre les deux versants de la langue arabe : écrit et oral, savant/religieux et populaire, en développant l'usage d'un arabe dit moderne aujourd'hui largement employé par les médias. La vénération et la fascination portées par les musulmans au texte de la Révélation ont conféré à la langue arabe un statut privilégié. Les Marocains éprouvent pour la fus'ha un grand respect et un attachement fort. Ils ont de fait développé une certaine exigence par rapport à la langue arabe classique.

Partant, la langue française représente pour de nombreux auteurs et journalistes marocains la langue de la «liberté », notion qu'ils emploient eux-mêmes ${ }^{12}$. Ils déclarent ne pas parvenir à se représenter la fus'ha comme une langue de l'intime, la considérant plutôt comme une langue chargée d'une valeur religieuse dans laquelle

10. Nicolas Vatin, Oral et écrit dans le monde turco-ottoman, Revue des mondes musulmans et de la Méditerranée, 75-76, 1996. 1998.

11. Éliézer Ben-Yehouda, Le Rêve traversé, Paris, Desclée de Brouwer,

12. Dominique Caubet, Les Mots du bled, Paris, L'Harmattan, 2004. 
ils éprouvent des difficultés à exprimer leurs sentiments et leurs idées propres, tant à l'écrit qu'à l'oral. Beaucoup de Marocains, écrivains ou non, expliquent ne pas réussir à dire «je t'aime» en arabe, préférant pour ce registre user du français. Du reste, les types d'amour sont linguistiquement différenciés dans la langue arabe qui offre un terme pour chaque état amoureux, une catégorisation qui complique la tâche à celui qui désire cacher la portée de ses sentiments. La précision offerte par la langue arabe ne permet pas de l'utiliser comme langue de la dissimulation. Le déplacement d'une langue à l'autre, en l'occurrence de l'arabe au français, ouvre alors un espace d'expression voilée. L'utilisation de la langue étrangère facilite, du moins rend possible la transgression des tabous, via l'usage $\mathrm{du} \| \mathrm{je} »^{13}$ et une prise de parole en son nom propre. Selon Karim Boukhari, rédacteur en chef de Tel Quel, les directeurs de publication des plus grands quotidiens et hebdomadaires marocains réunis à la suite de « l'affaire » Nichane ont estimé que le magazine était allé trop loin. Mais selon le directeur de La Vie économique: «Si le numéro était paru en français, ça ne se serait pas passé de cette manière. »

Selon certains auteurs et lecteurs assidus, le statut de la langue arabe et celui de la langue française auraient contribué à inscrire cette dernière dans un registre littéraire tourné vers l'individu, tandis que l'arabe exprimerait davantage le collectif ${ }^{14}$. L'usage écrit de l'arabe et du français ne relève en aucun cas d'une opposition entre tradition et modernité. La tension semble plutôt résider dans les liens existant entre liberté et contrainte. Le statut du français permet de l'utiliser pour exprimer ce qu'on ne peut pas ou que l'on n'a

13. La littérature de fiction et la poésie ont toujours constitué un espace d'expression personnel pour les auteurs marocains d'expression arabe. C'est seulement ces dernières années qu'ils commencent à publier des textes autobiographiques et à utiliser l'écrit comme un lieu propice à l'emploi du « je ».

14. Toutefois, un rapprochement a lieu ces dernières années entre pratiques d'écriture en langue arabe et en langue française, qui entraîne une redéfinition des dialectiques arabe/contrainte, français/liberté dont les frontières deviennent de plus en plus poreuses. Ce phénomène est rendu compte par l'émergence d'auteurs hommes et femmes d'expression arabe qui participent de plus en plus à l'élan littéraire du «parler vrai et identitaire » caractéristique de la littérature marocaine contemporaine. Même s'il s'agit là, en arabe, d'un genre littéraire encore en gestation, son émergence indique qu'au côté d'une écriture arabe classique, surtout représentée par la poésie, apparaît une écriture du soi, locale, marocaine. 
pas l'habitude de dire ou d'écrire dans sa langue maternelle ${ }^{15}$ l'arabe - comme cela a été le cas dans l'affaire des "noukâtes » («blague » en arabe). Toutefois, il ne s'agit pas là d'une caractéristique ontologique de la fus'ha qui ne représente pas en ellemême une langue sacrée. La stricte relégation de l'arabe classique à l'écrit et l'absence de son usage au quotidien ont placé cette langue dans une situation d'isolement qui a entraîné sa méconnaissance. Rares, en effet, sont les Marocains à avoir lu ou à avoir eu accès aux œuvres littéraires issues de la période anté-islamique qui ont, pour beaucoup d'entre elles, consisté à écrire l'intime et le désir ou encore à développer un questionnement critique sur l'Islam. Un constat renforcé par le fait que la fus'ha est, au Maroc, attachée à certains registres liés au pouvoir : la religion (dînn) et le droit religieux (fiqh), constituent deux domaines dans lesquels le protectorat français l'a cantonnée. C'est là un confinement qui en a fait la langue de la norme, liée au pouvoir et réservée à un petit nombre d'individus formant l'élite, détentrice de certaines connaissances transmises par voie écrite. Une particularité qui renforce son caractère tabou, garant de la hiérarchie socioculturelle immanente à la langue arabe, suivant laquelle les hommes de pouvoir ont accès à la langue écrite, tandis que les gens du peuple ne connaissent que l'arabe parlé.

\section{Dissimuler la subversion}

Par rapport à l'arabe classique, l'arabe dialectal (darija) et le berbère (amazigh) constituent les langues dans lesquelles les Marocains s'expriment spontanément. Cependant, ni la darija ni l'amazigh ne bénéficient d'un statut clair au Maroc si ce n'est en se glissant dans l'arabe officiel. Les pouvoirs en place essaient par tous les moyens de promouvoir la notion d'unicité, en particulier dans le domaine linguistique. Le monolithisme affiché dans les pays arabes, et particulièrement dans ceux du Maghreb, destiné à promouvoir les processus de création nationale et à les ancrer au sein d'une culture et d'une identité panarabe, a fait que cette pluralité linguistique a

15. Abdelfattah Kilito, La Langue d'Adam, Casablanca, Toubkal, 1999; Id., Tu ne parleras pas ma langue, Paris, Actes Sud, 2008. Kacem Basfao, « La littérature maghrébine : une question de langue », Annuaire de l'Afrique du Nord, 24/1987, éditions du CNRS, p. 379-384. 
longtemps été stigmatisée. Dans ce cadre, la diversité linguistique apparait comme éminemment transgressive, accusée de véhiculer des valeurs antinationales. C'est pourquoi l'État marocain cherche à restreindre l'emploi de ces langues en interdisant leur introduction dans la sphère publique ${ }^{16}$. Ceci pose un certain nombre de difficultés notamment du point de vue de l'alphabétisation des Marocains qui lisent dans une langue différente de celle dans laquelle ils parlent. En créant un magazine hebdomadaire écrit en arabe classique et en darija, tout l'enjeu d'Ahmed Benchemsi, directeur trentenaire de Nichane, est de sortir la darija du «ghetto linguistique où l'a enfermée la pensée officielle», en proposant aux Marocains un " magazine de proximité ». La singularité de Nichane est de traiter en darija de sujets de société de premier plan qui concernent et interrogent les Marocains : tels que la sexualité, la religion, la monarchie chérifienne ou la place de la femme. Peu de journaux, encore moins arabophones, jouent un tel rôle au Maroc. Une spécificité qui se manifeste notamment par le caractère esthétique de Nichane qui constitue le seul hebdomadaire arabophone sous format magazine en papier glacé, entièrement en couleur, à l'inverse des autres journaux qui sont des tabloïds (papier ordinaire) en noir et blanc.

Avant Nichane, Ahmed Benchemsi créa en 1999 le magazine Tel Quel, dont l'ambition était de dire le Maroc «tel qu'il est», sans craindre d'aborder les questions interdites : une première au Maroc. L'apparition de Tel Quel a suivi de près l'avènement de Mohammed VI en 1999, qui a favorisé l'émergence d'une nouvelle ère de liberté d'expression après le régime d'Hassan II. À cette époque, Ahmed Benchemsi estimait la société marocaine urbaine encore trop conservatrice pour recevoir et accepter en arabe des analyses sociétales allant à l'encontre de l'ordre établi. Lui et ses journalistes ont alors pris le parti de commencer par fonder un magazine francophone, s'adressant à la seule élite francophone, dans la perspective de créer ensuite son pendant arabophone ${ }^{17}$. Ce qui arriva

16. Sur ce point, voir l'article de Stéphanie Pouessel, «Écrire la langue berbère au royaume de Mohamed VI : les enjeux politiques et identitaires du tifinagh au Maroc », Langue, religion et modernité dans l'espace musulman, Revue des mondes musulmans et de la Méditerranée, 124/2008.

17. Ahmed Benchemsi et son équipe sont issus d'un milieu francophone, ayant pour la plupart entrepris leur formation à la Mission Française et à l'étranger. De ce fait, ils manipulent mieux la langue française que la langue arabe. Leur choix linguistique relève de leur parcours scolaire et universitaire et des représen- 
en octobre 2006, avec la parution de Nichane dont le titre, qui signifie « direct » en darija, renvoie à la ligne éditoriale de Tel Quel. À une chose près : Nichane est reçu par un public différent et surtout plus large incluant non seulement les élites arabophones, mais aussi les classes populaires. Les lignes à ne pas franchir, que constituent la religion, la monarchie et le Sahara occidenta $1^{18}$, sont fixées par le Makhzen - nom donné à l'État marocain - qui en assure le respect et le contrôle en s'efforçant de faire régner, sur elles, le silence. Après l'avènement au pouvoir de Mohammed VI, s'est opéré un certain brouillage des frontières. Les premiers journaux à avoir tenté de dépasser ces limites sont deux hebdomadaires francophones, $\mathrm{Tel}$ Quel et Le Journal dont les journalistes ont pu parler du roi ou de la religion de façon critique sans être réprimandés de manière systématique. Cependant, l'écart entre ce qui doit être tenu secret et ce qui peut être révélé est devenu presque imperceptible. Du jour au lendemain, l'attitude des autorités oscille entre mansuétude et rigorisme. L'arbitraire domine. Selon Driss Ksikés : «Au moment de Hassan II, les lignes rouges étaient claires et infranchissables. Depuis le début du règne de Mohammed VI, le flou règne, on sait qu'elles existent mais on ne sait pas où elles se situent, on le comprend après coup, seulement une fois qu'on les a dépassées. » Une observation partagée par Ahmed Benchemsi selon qui « la liberté d'expression ressemble aujourd'hui au Maroc à une cavalcade dans un champ

tations attribuées à la langue française. Selon M. Chentoufi, critique littéraire : «Beaucoup d'éditeurs et de libraires ont opté pour le français par dénigrement de la langue arabe », qui est associée au mieux au nationalisme, au pire à l'intégrisme. La permanence d'un public maghrébin francophone exprime la demande des nouvelles générations bilingues pour lesquelles l'attrait de la culture française s'exerce concurremment à celui de la culture et de la langue arabe. En outre, on attribue au français des valeurs proches de la liberté et de l'humanisme. La langue française évoque un certain degré d'engagement.

18. Le Sahara occidental est un territoire du nord-ouest de l'Afrique, bordé par le Maroc au nord, l'Algérie au nord-est, la Mauritanie à l'est et au sud, tandis que sa côte ouest donne sur l'Atlantique. Territoire non autonome selon l'ONU, cette ancienne colonie espagnole n'a toujours pas trouvé de statut définitif sur le plan juridique, plus de trente ans après le départ des Espagnols en 1976. Le territoire est revendiqué à la fois par le Maroc et par la République arabe sahraouie démocratique (RASD), fondée par le Front Polisario en 1976. Ce dernier mouvement, soutenu militairement, financièrement et diplomatiquement par l'Algérie, a pour objectif l'indépendance totale du Sahara occidental. Depuis le cessez-le-feu de 1991, le Maroc contrôle et administre environ $80 \%$ du territoire, tandis que le Front Polisario contrôle $20 \%$ de ce même territoire que le Maroc maintient derrière une longue ceinture de sécurité. 
de mines ${ }^{19} \gg$. On peut se demander quelles initiatives le Makhzen déploie pour affaiblir et contrarier les projets éditoriaux et quelles manœuvres opèrent les journalistes pour les déjouer. Cette situation instable a poussé les journalistes de Tel Quel et, aujourd'hui, de Nichane à mettre au point un certain nombre de stratégies dont la principale réside dans l'humour et la dissimulation: «Briser des tabous est notre objectif mais nous souhaitons le faire non pas sur un ton frontal et guerrier, plutôt sur un ton calme et sûr de soi, faisant une large place à l'humour. Nous débusquons les contradictions de notre vie politique et sociale, nous les mettons en évidence, et nous les laissons parler d'elles-mêmes, assorties d'un zeste d'ironie. Le message passe toujours mieux ainsi, et ceux que ça énerve y réfléchissent à deux fois avant d'attaquer. On est toujours un peu ridicule de s'en prendre à l'humour. " Le recours à la blague est l'exemple même de cette stratégie de dissimulation. La plaisanterie qui constitue le lieu privilégié de la subversion est sujette à la censure, et cette dernière est davantage contournée en français qu'en arabe. Ce phénomène est à relier non seulement aux représentations et aux pratiques attachées aux langues arabe et française que nous avons évoquées, mais aussi à la question essentielle de la réception du texte : dans quelle mesure la réception de l'écrit diffère en fonction du lectorat arabophone et francophone? Les écrits arabes et français sont-ils confrontés aux mêmes instances d'autorité?

\section{DU RAPPORT DU TEXTE À L'AUTORITÉ POPULAIRE}

\section{Une question de réception}

$\mathrm{Au}$ Maroc, la question de la cible joue un rôle important dans le choix des langues à utiliser pour évoquer les questions taboues. L'écart linguistique relève notamment du public, première autorité à laquelle l'écrit est confronté. Selon un journaliste : « Il n'y a qu'à regarder la télévision et lire la presse pour comprendre que, selon

19. Ces dernières années, l'État marocain exerce une pression forte sur les journalistes donnant lieu à des poursuites judiciaires qui contraignent les organes de presse à payer des amendes. Ceci pour leur rappeler que les lignes rouges à ne pas franchir sont toujours d'actualité. La presse marocaine, l'une des plus libres du monde arabe, est actuellement la cible d'un rappel à l'ordre très ostensible. 
la langue d'expression, arabe/français, on ne montre ni on ne dit la même chose puisque en tant que journaliste, la première préoccupation que nous avons est le public auquel nous nous adressons. » Les lecteurs arabophones et francophones appartiennent à des milieux sociaux distincts ${ }^{20}$. Le lectorat francophone se compose de personnes issues de classes aisées ayant réalisé leurs études à la Mission Française et à l'étranger. C'est pourquoi la langue française constitue le signe d'une ouverture vers l'extérieur qui véhicule une autre culture que celle strictement arabo-musulmane ${ }^{21}$. Dans l'anthropologie structurale, Lévi-Strauss considérait « le langage à la fois comme le fait culturel par excellence et celui par l'intermédiaire duquel toutes les formes de la vie sociale s'établissent et se perpétuent ${ }^{22} »$. Ainsi, chaque langue charrie la culture à laquelle elle correspond. La langue française est associée à des valeurs, des idéaux et des attentes différents de la langue arabe. Éloignée de la religion et de la Révélation, elle est plus proche des valeurs du siècle des Lumières : liberté, tolérance, laïcité ${ }^{23}$. Notons ici que le bilinguisme est rare en matière de lecture et d'écriture à Casablanca et à Rabat. Il s'agit donc bien de deux types d'écrits, tournés vers deux publics qui correspondent à des projets culturels pouvant être concurrents.

Le lectorat arabophone se compose quant à lui de Marocains issus de milieux populaires et bourgeois, qui présentent un attachement plus grand à l'Islam et au roi, sujets par rapport auxquels ils ont une position moins critique que le lectorat francophone. Une caractéristique à mettre en relation ces dernières années avec l'influence exercée par les musulmans extrémistes qui détiennent de plus en plus le monopole de la moralisation de la vie publique au Maroc. Cette position de domination repose notamment sur la création d'associations caritatives, par le biais desquelles ils exercent une pression conséquente sur la société marocaine urbaine à laquelle

20. Il existe deux lecteurs arabophones pour un lecteur francophone, un ratio qui, ces dernières années, tend à évoluer en faveur de l'arabe : Abdelali El Yazami, Enquête sur la lecture au Maroc, Rabat, Association marocaine des professionnels du livre, 1998.

21. De plus, les personnes qui lisent en français sont aussi celles qui regardent les émissions françaises par le biais de la télévision câblée.

22. Claude Lévi-Strauss, Anthropologie structurale, Paris, Plon, 1974, p. 392.

23. Propos recueillis lors de la présentation à Casablanca de l'ouvrage de Mohammed Arkoun, Humanisme et Islam, Paris, Vrin, 2005. 
ils tentent d'imposer un Islam rigoriste. Ceci porte atteinte au libre arbitre et au choix personnel de lecture, à l'instar de «l'affaire" des noukâtes, dont une a fait l'objet de vives condamnations. Voici le contenu de la boutade : Mohammed VI, roi du Maroc, se rend, le jour de sa mort, face à Dieu et lui demande où se trouve son père, Hassan II. Afin de faciliter la recherche, Mohammed VI lui remet la carte nationale de son père, mais Dieu ne trouve Hassan II ni au paradis, ni en enfer. La blague s'achève ainsi, signifiant qu'Hassan II, l'ancien roi du Maroc, n'est nulle part dans l'au-delà, tel un sous-homme, "tel un chien » me dit un lecteur de Nichane, Mohammed, âgé de quarante ans et employé dans une maison d'édition. Cette analogie n'est pas la seule transgression que comporte la noukâte : le fait que Mohammed VI s'adresse directement à Dieu a été pris par les musulmans rigoristes comme un véritable blasphème. Quelques jours après la sortie du numéro, les animateurs d'un site web islamiste portent plainte contre Nichane pour avoir "gravement offensé Dieu et son prophète ». Sur la centaine de commentaires postés sur le forum par des membres de la société civile, une large majorité traite les journalistes de l'hebdomadaire d'apostats et d'athées. Plus tard, le syndicat étudiant d'une faculté contrôlé par les « islamistes », tels qu'ils sont identifiés, organise un piquet de grève, distribue des affiches condamnatoires et appelle à la punition du «crime » commis par les journalistes. Le dossier des blagues fait l'objet d'un détournement opéré par des fondamentalistes soucieux de faire entendre avec force leur propre interprétation des noukâtes. Plus tard, une instance religieuse koweïtienne se saisit de l'affaire et lance un appel à la fatwa contre le magazine. La police est immédiatement sollicitée afin d'assurer la protection des journalistes et des locaux du magazine. Par la suite, le directeur de Nichane, Ahmed Benchemsi, répond à une convocation de la police judiciaire qui se solde par l'interdiction du magazine et la condamnation de deux journalistes.

Trois faits sont ici à relever: d'une part, le caractère immédiat de la réaction islamiste et ses répercussions dans la société; d'autre part, l'organisation des réseaux fondamentalistes notamment fondée sur des moyens technologiques performants pour diffuser l'appel à la condamnation; enfin, le caractère transnational de l'affaire avec l'intervention du Koweït. Comme l'a bien montré Jeanne Favret-Saada à propos des «caricatures de Mahomet », là 
aussi, les nouveaux médias ont contribué à «l'internationalisation de l'affaire» et à la production d'une «crise mondiale ${ }^{24} »$. Ces trois paramètres n'auraient pas été mobilisés, ou dans une moindre mesure, si l'article avait été écrit en français. Dans ce cas, en effet, Nichane n'aurait probablement pas fait l'objet d'une manipulation à des fins religieuses. Selon Driss Ksikés, cette affaire a révélé « que les clients de la liberté en arabe n'existent pas », encore trop soumis aux doctrines conservatrices, bien que leur émergence constitue tout l'enjeu du magazine. Un pari en bonne voie d'être tenu, précise Ahmed Benchemsi, puisque Nichane se hisse au deuxième rang des hebdomadaires arabophones avec 20000 exemplaires vendus en moyenne par semaine en 2007, talonnant le numéro 1 tirant à 24000 exemplaires et distanciant le numéro 3 plafonnant alors à 9000 exemplaires. Un signe indéniable que le magazine a un public important. Néanmoins, pour Ahmed Benchemsi :

Cette affaire nous a appris qu'il y a plus de conservateurs arabophones que de conservateurs francophones, donc plus de chances chez les premiers de tomber sur des populistes prêts à monter une affaire en épingle pour servir leur agenda politique personnel. À présent, nous faisons plus attention au choix des mots dès qu'il est question de religion ou de sexe, dans Nichane.

\section{Une double autorité}

Un quatrième fait est à signaler dans cette « affaire » : le dossier des noukâtes n'a fait l'objet d'une condamnation officielle qu'une semaine après avoir entraîné de vives réactions populaires. C'est la première fois qu'une fraction du peuple relaie l'État en jouant le rôle de censeur public. Un fait nouveau qui relève du caractère singulier et inédit de Nichane auquel la société marocaine dans son ensemble n'est pas encore habituée. Ainsi, celui-ci, davantage que Tel Quel, doit faire face à une autorité double - État/peuple - qui modifie et déplace la réaction par rapport au texte et à sa condamnation.

L'intervention de l'État dans le cadre de cette affaire a surtout consisté à contenir la réaction d'un autre agresseur : la part conservatrice de la société. On peut déceler dans la réponse du pouvoir le signe d'une inquiétude à l'approche des élections législatives à

24. Jeanne Favret-Saada, Comment produire une crise mondiale avec douze petits dessins, Paris, Les Prairies ordinaires, 2007, p. 79. 
l'issue desquelles l'État craint que le Parti islamiste de la justice et du développement (PJD) devienne la première formation politique au Parlement. Le Makhzen s'est positionné de façon claire et tranchée dans cette affaire pour signifier qu'il ne manquait pas à son rôle de garant du respect de l'Islam. Un prétexte qui aurait pu alimenter la campagne politique du PJD, déjà ciblée sur le recul des valeurs religieuses dans un pays considéré comme laxiste. Selon Karim Boukhari, l'intervention de l'État a permis de calmer les tensions, sans quoi la réaction aurait été, selon lui, plus grande : le peuple mené par les extrémistes, considérés par les journalistes de Tel Quel et de Nichane comme « islamistes », se serait employé seul à faire régner la justice fondée sur le respect de la Charia. L'État a alors joué le rôle de garde-fou. C'est la première fois qu'une telle ambivalence de fonction apparaît de façon aussi forte : l'autorité makhzénienne représente à la fois une rivale et un allié pour les journalistes de Nichane. Karim Boukhari explique cela clairement :

À la fois l'État te mate, tel est son rôle, s'il ne le fait pas, on le lui reprochera, et, à la fois, il te protège, même s'il te sanctionne : en te tapant un peu, il t'évite le risque de te faire taper plus fort par ton voisin, et là tu crains pour ta vie [...] C'est une espèce d'évolution bizarre et inattendue, d'abord, on a vu l'État en tant qu'adversaire, en tant que "autre", mais maintenant, on apprend à le considérer comme un partenaire... et ça, c'est nouveau. C'est apparu avec Nichane ${ }^{25}$.

Ainsi l'autorité, face à laquelle sont habituellement déployés des systèmes de défense visant à contourner l'interdit, devient protection. Nichane et le Makhzen partagent une lutte commune : empêcher la montée des islamistes au pouvoir. Un lien de solidarité les unit dans l'«affaire» des noukâtes, étant tous les deux tributaires de l'opinion populaire. Karim Boukhari affirme : « Je préfère choquer un haut responsable, quitte à ce qu'il soit le roi, plutôt que de choquer mon voisin. Avec le premier, je peux parler, je peux dealer, mais pas avec un forcené. Tu ne sais pas de quoi sa tête est faite». Le rédacteur en chef de Tel Quel exprime ici la tyrannie exercée par une fraction de la société marocaine sur les écrits en arabe et la manière dont celle-ci conditionne l'écriture. Cette situation génère un phénomène d'autocensure plus important en arabe qu'en français, confinant une fois de plus la première langue dans le silence.

25. Karim Boukhari, 4 mars 2008. 


\section{L'usage de la darija, acte de transgression?}

L'emploi de la darija, langue de communication nationale, comme langue d'écriture, a également faitl'objet de sévères condamnations de la part des autorités ayant interrogé Ahmed Benchemsi. Cette situation pose la question de savoir si ce qui est entendu peut être lu, cela amenant à interroger le statut de l'oralité et de l'écriture dans la société marocaine, dite de tradition orale ${ }^{26}$. Dans ce type de société, l'écrit revêt un statut particulier lié au pouvoir : seule l'élite sociale, religieuse et politique y a accès ${ }^{27}$. Il regroupe un ensemble de connaissances réservées à quelques-uns. De ce rapport à l'écrit naît souvent une dévalorisation de la tradition orale dont l'indignité est perçue au Maroc à travers un enjeu essentiellement religieux - la maîtrise de la langue arabe étant liée à la connaissance du Coran ${ }^{28}$. Cette partition de la langue - langue savante/langue du peuple -, a entraîné une scission entre les classes sociales, scission s'estompant avec l'alphabétisation. L'imprimé constitue un objet de savoir précieux et puissant, qui devient l'objet d'un enjeu politique fort, d'où son cantonnement à une étroite frange de la société2 ${ }^{2}$.

Via la création d'un magazine qui combine arabe classique et arabe dialectal, Ahmed Benchemsi a cherché à réunir les deux lectorats qui leur correspondent, bien qu'ils occupent une position hiérarchique différente au sein de la société. Le projet de Nichane répond alors à une ambition claire, à savoir « réconcilier » les Marocains avec leur identité :

Les Marocains parlent en darija et écrivent en arabe classique. C'est une diglossie, pour ne pas dire un indicateur de schizophrénie, contre lequel nous nous sommes toujours élevés et contre lequel nous entendions nous lever, de manière pratique cette fois, dans Nichane. Et cela, partant d'une conviction simple : nous réconcilier avec notre langue est le premier pas pour nous réconcilier avec notre identité, donc avec nous-mêmes.

Nichane donne aux classes populaires la possibilité d'interroger, dans leur langue, les questions taboues - comme l'Islam et la poli-

26. Pascal Boyer, « Orale (tradition)», Paris, Encyclopedia Universalis, Corpus 6, Paris, France S. A., 1995.

27. Collectif, Langues et cultures au Maghreb, Revue Prologues, 17/2002.

28. Jocelyne Dakhlia, L'Oubli de la cité, Paris, La Découverte, 1990.

29. N. Atiyeh, « The book in the modern arab world», in Id., The Book in the Islamic World, New York, 1995. 
tique - situées au fondement du pouvoir des élites qui cherchent à en avoir le contrôle. Combiner l'arabe dialectal et l'arabe classique, soit confondre les deux classes sociales que ces deux langues représentent pour tenter d'unifier l'identité marocaine, constitue un acte transgressif. En effet, celui-ci fait accéder le peuple à un ensemble de connaissances "secrètes", jusque-là strictement relégué à une fraction de la société. Imposer la darija dans sa forme écrite, et installer la parole populaire dans un champ textuel normalement réservé à l'expression du Makhzen, revient à porter atteinte à cette division sociale du pouvoir. Cette transposition de l'oralité à l'écriture élève le peuple au rang de l'élite, faisant émerger l'autorité populaire.

Après l'interdiction de Nichane pendant près de deux mois, celui-ci reparut en février 2007 et fit l'objet quelques mois plus tard d'une autre «affaire». En août 2007, Ahmed Benchemsi a été inculpé de «manquement au respect dû à la personne du roi » pour avoir écrit une analyse critique du discours du trône, prononcé par Mohammed VI le 30 juillet précédent. Cette analyse avait fait l'objet d'un éditorial en français et en arabe dans les numéros de $\mathrm{Tel}$ Quel et de Nichane parus la même semaine. Là encore, la version française n'a pas posé de problèmes. Sous le titre Où tu m'emmènes mon frère?, en référence à une célèbre chanson du groupe Nass el Ghiwane sur le malaise de la jeunesse marocaine des années 1970, Benchemsi interpellait directement le souverain, lui reprochant, en substance, de cumuler trop de pouvoirs tout en se posant indûment en garant de la démocratie au Maroc. Ce n'est pas tant le fond de son éditorial que la forme qui a valu à Benchemsi d'être interrogé pendant vingt heures par la police, puis assigné en justice, tandis que ses deux revues étaient saisies et pilonnées. Une fois encore, en utilisant la darija, langage sans détour, presque cru, qui reprend les expressions de la rue et des cafés, le journaliste avait commis un acte transgressif du fait qu'il s'adressait à son souverain comme à son frère ou à son voisin : « J'ai donné l'impression d'avoir oublié l'étiquette alors que je m'adressais au roi! Je lui ai parlé comme à un homme, lui, le "commandeur des croyants"! »

L'écriture, en effet, n'est pas seulement une forme de représentation du savoir, elle en détermine aussi le contenu, et présente pour le Makhzen le risque que la darija change de statut. Passer de la langue du peuple à la langue savante impliquerait un nouveau mode 
de pouvoir. Selon Jack Goody, auteur d'une étude sur l'impact de l'écriture et le lien qui existe entre le mode de pensée et les moyens de pensée : « tout changement dans le système de communications a nécessairement d'importants effets sur le contenu transmis. Nous devons partir de ce fait que l'acquisition du langage, cet attribut spécifiquement humain, est à la base de toutes les institutions sociales, de tout comportement normatif ${ }^{30} »$. L'écrit agit donc : faire muter la langue, la darija, en la transposant à l'écrit a des incidences concrètes sur la société et les institutions autour desquelles elle s'organise. Écrire la langue populaire représente une transgression de la hiérarchie socioculturelle immanente à la langue arabe. En outre, le dossier de Nichane a consisté en la mise à l'écrit dans la langue du peuple de propos subversifs que l'écriture a permis d'officialiser et de faire exister. En recevant le sceau de l'imprimé la blague se voit attribuer un caractère officiel, à l'instar de tout ce qui est écrit dans les sociétés à tradition orale. Il s'agit là de la contravention d'une règle implicite consistant à laisser une marge de subversion à l'oral, dans le milieu privé, et non à l'écrit, qui symbolise l'espace public ${ }^{31}$. Afin d'enrayer ce mouvement de mutation de la langue, le Makhzen a tenté de récupérer les numéros invendus de Nichane chez les buralistes et les kiosquiers pour les détruire par le feu. Un autodafé dont l'objectif est de supprimer toutes traces d'un écrit subversif. Un acte qui se place au-delà de la censure sociale, dans la persécution étatique. Par la destruction, le Makhzen a voulu rappeler qu'il était le seul à avoir le contrôle de l'arabe, du savoir écrit produit dans cette langue, de son élaboration et de son officialisation. Certains sujets connus par les élites francophones ne peuvent pas être énoncés dans la langue du peuple. Ainsi, la diglossie de la langue arabe se trouve consolidée par une division supplémentaire, entre le silence auquel le Makhzen plie la langue arabe officielle et la parole libérée, principalement véhiculée par la darija, à l'oral.

30. Jacques Goody, La Raison graphique, Paris, Éditions de Minuit, 1979, p. 46-47.

31. Il est néanmoins important de noter que de plus en plus de groupes de musiciens ou d'émissions radiophoniques traitent en darija de sujets de société délicats, comme la religion ou la sexualité. 
Ainsi, le secret sur les sujets politiques et religieux est bien affaire de langues qui sont aux prises dans le Maroc urbain, à Rabat et à Casablanca, avec des questions de légitimité et d'autorité. Alors que la langue française autorise leur traitement, la langue arabe impose le silence. Dans cette perspective, l' "affaire Nichane»" illustre le poids des tabous linguistiques dans la société marocaine urbaine. Elle signale que pour ses acteurs, le jeu est double : du côté du Makhzen, la censure réprime autant qu'elle protège les journalistes; du côté de ces derniers, l'échec de l'usage de la darija, plutôt que de la langue française, comme langue de l'émancipation, confirme le statut ambigu de cette dernière au Maroc. Les journalistes s'appuient sur la censure elle-même, pour se protéger. Le paradoxe et la profonde ambivalence de la censure se donnent à voir au cœur de cette affaire.

Le scandale qu'elle engendre représente un moment de transformation sociale qui a pour action de réaffirmer et de renforcer les valeurs transgressées plutôt que d'opérer une démonstration collective de leur obsolescence. Suivant le sens analytique qu'Élisabeth Claverie $^{32}$ donne à la notion d'affaire comme « chose jugée au tribunal puis contestée de l'extérieur et proposée au jugement du public, lequel, rendant un jugement inverse, détachera les personnes de leur affectation initiale et les fera permuter ${ }^{33}$ ", l'affaire Nichane est « ratée ». En effet, le procès intenté contre Driss Ksikés n'a donné lieu à aucune requalification des acteurs : les victimes - Dieu et son représentant, le Roi - et les accusés - les journalistes du magazine n'ont pas changé de place. L'offensé est resté la société outragée dans sa croyance aux valeurs sacrées, et Driss Ksikès, le coupable. Quant au peuple, loin de s'être rangé du côté de l'accusé, il l'a acculé au jugement. Du reste, la sentence a rappelé et, par là, consolidé le caractère immuable et indiscutable du « blasphème » entendu ici comme la profération injurieuse contre «l'honneur de Dieu ${ }^{34} »$. Ce faisant, l'affaire Nichane n'a pas été l'occasion d'une mise à

32. Élisabeth Claverie, Denis Laborde, Philippe Roussin (dir.), Critique et affaires de blasphème à l'époque des Lumières, Paris, Honoré Champion, 1998, p. 7; Luc Boltanski, Élisabeth Claverie, Nicolas Offenstadt, Stéphane Van Damne (dir.), Affaires, scandales et grandes causes, Paris, Stock, 2007.

33. É. Claverie, D. Laborde, P. Roussin (dir.), Critique et affaires de blasphèmes, op. cit., p. 7.

34. L. Boltanski, É. Claverie, N. Offenstadt, S. Van Damne (dir.), Affaires, scandales et grandes causes, op. cit. 
l'épreuve de la connexion intime entre le religieux et le politique. Elle n'a pas non plus opéré de division entre les masses populaires et le Makhzen dont elle a plutôt célébré la communion. Si l'accusation de blasphème a permis de reconnaître une division dans la société marocaine urbaine entre progressistes et conservateurs, l'absence de défense a montré à quel point ces derniers dominent l'opinion publique. Encore aujourd'hui, il est plus répréhensible de traiter certains sujets en arabe, langue du pouvoir, qu'en français. L'affaire Nichane met donc en évidence la constitution problématique d'une opinion publique en marge des valeurs religieuses.

Elle souligne plus que de simples rapports de rivalité : elle montre que c'est une partie de la population qui ne souhaite pas entendre. Un constat qui implique d'élargir les dimensions du secret et du silence telles qu'elles ont été entendues dans le cadre de cette réflexion. Pour Sophie Foch Rémusat ${ }^{35}$, le secret est « une forme limite de communication, une forme qui n'est presque plus verbale, une parole soustraite à la circulation et à la monétarisation en signes du langage. Le secret se dépose sous forme de murmure dans le canal même de l'oreille : il est une caresse. L'enjeu est donc de trouver une voie d'accès à ceux qui, dans la société, désirent la vérité et la liberté. Ceux-là, ces écrivains, s'ils les aiment, doivent les baiser [sic] en inventant des fictions, des fables et des métaphores pour avoir l'air de parler d'autre chose à d'autres gens. Dire des choses toutes crues dans la langue que tout le monde entend, n'est peutêtre pas la voie de la liberté. Pour s'exprimer, celle-ci emprunte des détours, justement pour ne pas se laisser prendre à la perversion dont elle se méfie ».

anouk.cohen@wanadoo.fr

35. Je tiens à remercier Sophie Foch Rémusat, professeur de philosophie, qui a accepté de relire cette étude en me faisant part de ses remarques riches et pertinentes qui m'ont permis d'affiner l'analyse menée sur la notion du secret. 